\title{
New drugs to treat multidrug-resistant tuberculosis: the case for bedaquiline
}

REVIEW

This article was published in the following Dove Press journal:

Therapeutics and Clinical Risk Management

29 July 2014

Number of times this article has been viewed

Eric Leibert

Mauricio Danckers

William N Rom

Department of Internal Medicine, Division of Pulmonary, Critical Care and Sleep Medicine, New York University School of Medicine, New York, NY, USA
Abstract: Mycobacterium tuberculosis develops spontaneous resistance mutants to virtually every drug in use. Courses of therapy select for these mutants and drug-resistant organisms emerge. The development of drug-resistant organisms has reached the point that drug resistance now threatens to undermine global success against tuberculosis (TB). New drugs are needed. The last new class of drugs specifically developed for treatment of TB was the rifamycins over 40 years ago. New funding sources and the development of product development partnerships have energized the TB drug development effort. There are now more TB drugs in development than at any time in the past. The first of these drugs to be developed and marketed was bedaquiline. Bedaquiline has an entirely novel mechanism of action and so should be active against otherwise highly resistant organisms. It acts on the transmembrane component of adenosine triphosphate synthase and acts by preventing electron transport. This raises the exciting possibility that bedaquiline may be active against less metabolically active organisms. Drug-drug interactions between rifamycins and the cytochrome P450-3A system will limit bedaquiline's utility and create complexity in treatment regimens. In clinical trials, treatment with bedaquiline added to a background multidrug-resistant TB regimen was associated with earlier culture conversion and higher cure rates, but there were unexplained excess deaths in the bedaquiline arms of these trials. Food and Drug Administration approved bedaquiline for the treatment of multidrug-resistant TB when an effective treatment regimen cannot otherwise be provided. They required a black box warning about excess deaths and require that a phase III trial be completed. A planned Phase III trial is being reorganized. While bedaquiline is an exciting drug and marks a dramatic moment in the history of TB treatment, its ultimate place in the anti-TB drug armamentarium is unclear pending the Phase III trial and the development of other new drugs that are in the pipeline.

Keywords: bedaquiline, multidrug-resistant tuberculosis, Sirturo

\section{Introduction}

While currently available antituberculous medications are highly effective against susceptible strains ${ }^{1}$ and rates of tuberculosis have been declining steadily in developed countries, ${ }^{2}$ the majority of the global burden of tuberculosis is in resource-limited countries where tuberculosis, human immunodeficiency virus (HIV) coinfection and drug-resistant tuberculosis intersect. ${ }^{3}$

Rates of tuberculosis increased dramatically in the developing world in the 1980 s and 1990s, fueled by the HIV epidemic and by limited public health infrastructure. ${ }^{2}$ While rates have stabilized, they remain extremely high. ${ }^{4}$ Resources are not available for universal culture and susceptibility testing. Despite obvious dangers, ${ }^{5}$ empiric therapies have been employed based on assumptions of low rates of drug resistance. Reports of
New York University School of Medicine,

462 First Avenue, 7-N-24,

New York, NY, USA 10016

Tel +l 2122636479

Fax + I 2I2 2638442

Email eric.leibert@nyumc.org 
high rates of multidrug-resistant tuberculosis (MDR-TB) and extensively drug-resistant tuberculosis (XDR-TB) ${ }^{6,7}$ in recent years have highlighted the failure of that approach, the need for cheap and rapid diagnostic and susceptibility tests that can be made widely available, and the need for new drugs.

MDR-TB and XDR-TB are treated with drugs that are less effective, more expensive, more toxic, and that require longer courses of treatment than drugs used in the treatment of susceptible organisms. ${ }^{8,9}$ New drugs are needed.

\section{Toward a new drug for tuberculosis}

Rates of HIV infection are high across much of sub-Saharan Africa. During coinfection with Mycobacterium tuberculosis and HIV, each infecting organism potentiates the effects of the other. ${ }^{10,11}$ For patients with tuberculosis who are coinfected with HIV, early addition of antiretroviral therapy can be lifesaving. ${ }^{12-14}$ There are important interactions between rifamycins, antiretrovirals and the cytochrome P450-3A (CYP3A) system. Non-nucleoside reverse transcriptase inhibitors and rifamycins induce CYP3A. Non-nucleoside reverse transcriptase inhibitors and protease inhibitors are metabolized by CYP3A. ${ }^{15}$ These interactions add to complexity and risk in the treatment of coinfected individuals. ${ }^{16,17}$

When growing rapidly, $M$. tuberculosis organisms make cell wall lipids and synthesize protein. When subjected to stress, the organisms react by activating a series of metabolic changes including decrease in cell wall synthesis and shift in protein synthesis away from replication and toward survival functions. These changes, collectively referred to as the dormancy regulon are well adapted to allow survival of the organism intracellularly and under hypoxic and nutritionally limited conditions. ${ }^{18}$

Some drugs kill by interfering with cell wall synthesis. These may be highly effective, as for example, isoniazid (INH) is, at controlling active disease caused by rapidly dividing organisms, a quality measured by early bactericidal activity (EBA). ${ }^{19}$ Drugs that kill organisms attacking a different vital pathway might be better able to kill organisms whose metabolism has shifted into dormancy. Specifically, a drug that could target the cell's energy metabolism could be potent even against less metabolically active organisms. While an organism might remain alive while not making cell walls, and even while synthesizing very little protein, it cannot maintain an electrified cell membrane gradient and survive. ${ }^{20}$

It is estimated that one-third of the population is latently infected with M. tuberculosis. ${ }^{3}$ Any effective tuberculosis control strategy will have to include an approach to managing this reservoir of potential new cases of active disease. ${ }^{21}$
Since latent organisms are nevertheless alive, a new drug active against dormant organisms might also be able to kill latent organisms. This property would revolutionize tuberculosis control efforts.

Desirable characteristics of new drugs are well known. New drugs should be safe. They should be highly effective against both susceptible and resistant strains. Drugs with a novel mechanism of action are more likely to meet this requirement. New drugs should be affordable and orally available. They should not interact with existing antituberculous drugs or antiretrovirals. Half-lives of the drugs should permit pairing of drugs with similar half-lives so that inadvertent selective pressures can be avoided.

The emergence and funding of not-for-profit public partnerships for product development has revolutionized the landscape for the development of new antituberculous drugs. The Global Alliance for TB Drug Development has agreements with a variety of pharmaceutical companies to support discovery and development of new drugs and regimens. While costs remain high and the chances of any individual candidate drug succeeding are remote, ${ }^{22}$ there are more prospective antituberculous drugs in the pipeline now than ever before, ${ }^{23}$ representing potential breakthroughs in the treatment of both susceptible and drug-resistant tuberculosis.

\section{Bedaquiline Preclinical development}

Bedaquiline, trade name Sirturo, was developed as R207910 and later TMC207. In publications describing combination regimens, it is often abbreviated as "J", for Janssen Pharmaceuticals (Titusville, NJ, USA). Bedaquiline is a diarylquinoline. Diarylquinolines are related to, but mechanistically different from quinolones as they do not inhibit $M$. tuberculosis DNA gyrase. Bedaquiline links to the transmembrane domain of the adenosine triphosphate (ATP) synthase. This interferes with the mycobacterial conversion of adenosine diphosphate into ATP by interrupting transmembrane and central stalk rotation of the proton pump. This is a novel mode of action for antituberculous drugs. ${ }^{24}$ Bedaquiline is $2 \times 10^{4}$ more avid for mycobacterial than for mammalian ATP synthase. ${ }^{25}$ Bedaquiline has activity against many mycobacterial species. ${ }^{26}$

Bedaquiline is active against both susceptible and multidrug-resistant strains of $M$. tuberculosis as would be expected of a drug with a novel mechanism of action. ${ }^{27}$ Mutations in residues 63 and 66 of the transmembrane domain of the ATP synthase complex impede bedaquiline's access to its target at residue 61 , causing resistance. ${ }^{28}$ 
Spontaneous resistance mutations occur in one in $10^{7}$ to one in $10^{8}$ organisms, a rate comparable to rifampin. This resistance was attributed to mutations in the $a t p E$ gene. ${ }^{24}$ Drug efflux is another potential mechanism of resistance to bedaquiline. Minimal inhibitory concentrations for bedaquiline for M. tuberculosis clinical isolates fall when verapamil, a drug efflux inhibitor, is added. ${ }^{29}$ Clofazimine-resistant mutants have been investigated and found to also be resistant to bedaquiline. A mutation in $R v 0678$, a transcriptional repressor gene and associated upregulation of a MmpL5, multisubstrate efflux pump, accounted for this cross-resistance. ${ }^{30}$ Acquired resistance to bedaquiline has been observed in clinical trials ${ }^{31}$ and will certainly emerge as the drug is used in practice.

Bedaquiline has a large volume of distribution and a half-life of 43-64 hours in plasma and 28-92 hours in tissue (including lung and spleen). ${ }^{24}$ Bedaquiline's long terminal half-life of 4-5 months ${ }^{31}$ raises concerns that even after drug cessation, effective monotherapy may continue for months, potentially leading to bedaquiline resistance, and any adverse effect could be prolonged.

If half-lives are properly matched, a regimen of drugs with long half-lives has the potential to allow highly intermittent dosing. In a murine model, bedaquiline, rifapentine (which has a serum half-life of $14-18$ hours), ${ }^{15}$ and pyrazinamide (PZA) given once weekly outperformed a regimen of INH, rifampin, and PZA given five times per week over a 2-month period. . $^{32,33}$

\section{Clinical development}

Bedaquiline has good bioavailability following oral administration. ${ }^{34}$ Bioavailability increases by about two-fold with food. ${ }^{31}$ There is no need to adjust dose for patients with moderate renal or liver disease. Caution is recommended for those with severe renal or liver disease. ${ }^{35}$

Bedaquiline is metabolized by CYP3A4 to less active metabolites. ${ }^{31}$ CYP3A4 inducers or inhibitors may interfere with bedaquiline concentrations. ${ }^{36}$ Combination of bedaquiline with rifampin significantly reduces bedaquiline levels. Bedaquiline does not interact with INH, PZA, ethambutol, kanamycin, ofloxacin, or cycloserine. Bedaquiline can be safely administered with nevirapine without dosage adjustment, but dose adjustment may be needed for use with lopinavir plus ritonavir, and efavirenz. ${ }^{31}$

EBA is poor during the first several days. Bactericidal activity improves between weeks 1 and 4, suggesting a timedependent killing. ${ }^{35}$ Because bedaquiline's mechanism of action is interference with ATP synthesis, it is possible that intracellular ATP stores must be depleted before bactericidal activity occurs. Increasing doses of bedaquiline improves EBA. A loading dose of $400 \mathrm{mg}$ daily leads to higher bedaquiline concentrations and a 2-day EBA that is similar to the 14-day EBA. ${ }^{37}$ The recommended dose of bedaquiline is $400 \mathrm{mg}$ daily for 2 weeks, then $200 \mathrm{mg}$ three times per week for the duration of treatment. Bedaquiline has excellent late bactericidal activity, suggesting that it may kill nonreplicating organisms. This property could potentially allow for shorter treatment durations..$^{27,38}$

\section{Phase II trials}

Efficacy of bedaquiline has been assessed in randomized placebo-controlled trials and in an open-label single-arm observational study. Data for all bedaquiline studies is compiled in the publicly available US Food and Drug Administration (FDA) briefing. ${ }^{31}$ C208 stage 1 was a randomized, multicenter, double-blind, placebo-controlled trial to assess safety and efficacy of 8 weeks of bedaquiline in subjects with MDR-TB. ${ }^{35,39}$ Exclusion criteria included prior treatment for MDR-TB, fluoroquinolone or injectable resistance, significant cardiac arrhythmia, history of alcohol abuse, and advanced HIV disease (CD4 $<300$ cells $/ \mathrm{mm}^{3}$ ). Forty-seven participants with new onset pulmonary MDR-TB received a five-drug second-line background regimen of kanamycin, ofloxacin, ethionamide, PZA, and cycloserine or terizidone and were randomized to bedaquiline versus placebo for the first 8 weeks of therapy. The dose of bedaquiline was $400 \mathrm{mg}$ daily for 2 weeks then $200 \mathrm{mg}$ three times a week for 6 weeks. This dosing was chosen to maximize EBA, then to maintain a serum concentration of $600 \mathrm{ng} / \mathrm{mL} .{ }^{34}$ After 8 weeks of treatment, participants remained on the background regimen for 24 weeks. Time to culture conversion was shorter for subjects taking bedaquiline than for those taking placebo. At 8 weeks, the culture conversion rate in the bedaquiline group was $48 \%$ compared with $9 \%$ in the placebo group. At 24 weeks, culture conversion was $81 \%$ in the bedaquiline group and $65 \%$ in the control group. . $^{31,35,39}$

The second stage of $\mathrm{C} 208$ was similar in design to the first stage, but continued bedaquiline for 24 weeks and followed subjects for 96 weeks. Bedaquiline or placebo was added to a standard treatment regimen for MDR-TB. Eighty subjects received bedaquiline and 81 subjects received placebo. The median time to sputum conversion was 83 days in the bedaquiline group and 125 days in the control group. At week 24, the sputum conversion rate was $79 \%$ in the bedaquiline group and 58\% in the control group. ${ }^{31}$ In both stages of C208, fewer subjects in the bedaquiline arm than in the control arm developed pre-XDR-TB (resistance to either a fluoroquinolone or 
a second-line injectable drug) or XDR-TB (one versus four in C208 stage 1 and zero versus seven in C208 stage 2). ${ }^{31}$

Study C209 was a Phase II single-arm, open-label study of bedaquiline in 233 subjects with MDR-TB, pre-XDR-TB, and XDR-TB (but with an available three-drug background regimen). People with severe extrapulmonary tuberculosis, HIV with CD4+ counts below 250 cells $/ \mathrm{mm}^{3}$, and those receiving incompatible antiretrovirals were excluded. Subjects were treated with 24 weeks of bedaquiline added to a background regimen of 18 to 24 months. Five percent of subjects were HIV positive. Fifty-four percent had MDR-TB; $25 \%$ had pre-XDR-TB; and $21 \%$ had XDR-TB. In subjects with MDR-TB, the median time to culture conversion was 8 weeks, and the conversion rate at week 24 was $87.1 \%$. In subjects with pre-XDR-TB, the median time to culture conversion was 12 weeks, and the conversion rate at week 24 was $77.3 \%$. In subjects with XDR-TB, the median time to culture conversion was 24 weeks, and the conversion rate at week 24 was $55.6 \%$. Conversion rates were higher in PZA-susceptible subjects and in those whose background regimen included three or more drugs active against their organism. ${ }^{31,40}$

Treatment-emergent bedaquiline resistance was observed. Twenty-eight subjects in C208 and C209 had pairs of isolates from before and after bedaquiline treatment. Ten of these had increases in bedaquiline minimal inhibitory concentration. Development of resistance occurred more commonly in subjects with XDR-TB than MDR-TB, suggesting prevention of selective pressure by more robust regimens. ${ }^{31}$

Bedaquiline causes corrected QT interval (QTc) prolongation with a mean increase of around $15 \mathrm{~ms}$. Among 44 volunteers in C1003, 102 in C208, and 233 in C209, two had QTc over $500 \mathrm{~ms}$ and 19 had increases over baseline of $60 \mathrm{~ms}$. Combination with clofazimine and ketoconazole increases this effect. ${ }^{31}$

Nausea, arthralgia, headache, hyperuricemia, and emesis are the most common adverse events. Drug-related hepatic disorders were more common in subjects taking bedaquiline $(8.8 \%)$ than in those receiving placebo $(1.9 \%)$. Most were elevated transaminases. ${ }^{31}$

In C208 stage 2, ten of 79 bedaquiline-treated subjects died compared with two of 81 placebo-treated subjects. Five bedaquiline-treated subjects died of tuberculosis. Other causes of death were myocardial infarction, alcohol poisoning, hepatitis with cirrhosis, peritonitis with septic shock, cerebrovascular accident, and motor vehicle accident. There was a wide range in time from last dose of bedaquiline to death, 2 to 911 days. All but one of the deaths occurred after the subject had completed the bedaquiline component of treatment. No death in the bedaquiline arm was considered related to the study drug by the investigators. There was no relationship between QTc prolongation and survival. ${ }^{31}$

In C209, 16 subjects had died by the July 15, 2012 cut-off date, 12 during the study and four after discontinuation of study drug. Nine of these deaths were related to tuberculosis. One of these deaths, in a subject who developed renal failure after emesis and dehydration, was considered potentially related to bedaquiline by the investigators. Other causes of death included lung infection, cor pulmonale, pyopneumothorax with respiratory failure, cardiac arrest, hemoptysis, and hypertension. None of these subjects had a treatment emergent QTc $\geq 500 \mathrm{~ms}^{31}$

\section{Regulatory approval}

On December 28, 2012, the US FDA approved bedaquiline for MDR-TB "when an effective treatment regimen cannot otherwise be provided". ${ }^{41}$ The approval was through an accelerated approval program that allows approval of a drug for a serious disease based on surrogate endpoints. ${ }^{42}$

The FDA advisory panel vote on efficacy was 18 to zero. The vote on safety was eleven to seven. Because of QTc prolongation and significant excess mortality, the US FDA required a boxed warning on the bedaquiline package insert: ${ }^{43}$

An increased risk for death was observed in the Sirturo treatment group $(9 / 79 ; 11.4 \%)$ compared with the placebo treatment group $(2 / 81 ; 2.5 \%)$ in one placebo-controlled trial. Only use Sirturo when an effective treatment regimen cannot otherwise be provided;

QTcF prolongation can occur with Sirturo. Use with drugs that prolong the QT interval might cause additive QTcF prolongation.

As a condition of the accelerated approval program, Janssen Therapeutics is required to enroll everyone started on bedaquiline in a patient registry to track adverse events and outcomes.

Janssen is also required to conduct a Phase III multicenter randomized double-blind placebo-controlled trial of bedaquiline in subjects with sputum-smear-positive pulmonary MDR-TB. C210 was to be that study and was initially scheduled to begin accruing subjects in 2013. C210 was to include 600 adults with smear-positive MDR or pre-XDR-TB. Bedaquiline or placebo was to be given with a background MDR-TB regimen for 36 weeks. Levofloxacin was to be the fluoroquinolone used in order to minimize QTc interactions. ${ }^{31}$ Subjects were to be followed for 132 weeks. The primary endpoint was to be favorable outcome at week 60. As of February 2014, C210 had been 
withdrawn prior to enrollment. ${ }^{44}$ Because a Phase III trial was a condition of approval, it is anticipated that such a trial will eventually be completed, though likely by different investigators and possibly by incorporating a bedaquiline arm into a larger project.

The Centers for Disease Control and Prevention (CDC) guidelines on use of bedaquiline were published in October 2013. Aspects of these guidelines go beyond current FDAapproved labeling for bedaquiline. CDC guidelines state that:

Bedaquiline may be used on a case-by-case basis in children, HIV-infected persons, pregnant women, persons with extrapulmonary MDR-TB, and patients with comorbid conditions on concomitant medications when an effective treatment regimen cannot otherwise be provided [and that] bedaquiline may be used on a case-by-case basis for durations longer than 24 weeks when an effective treatment regimen cannot be provided otherwise. ${ }^{45}$

\section{Discussion}

Bedaquiline's novel mechanism of action suggests that it should have activity against resistant organisms. Late bacterial activity and animal data suggest activity against dormant or nonreplicating bacilli that could allow shorter treatment courses. ${ }^{39}$

An important step will be to determine the compatibility of bedaquiline with the other candidate antituberculous drugs that are moving through the clinical development pipeline, particularly the nitroimidazoles delamanid (OPC-67683) and PA-824. These drugs, potentially in combination with PZA and moxifloxacin, could be important elements of new short-course regimens for susceptible and drug-resistant tuberculosis. ${ }^{46}$

Bedaquiline was approved based on trials that evaluated the surrogate marker of culture conversion rather than the gold standard of durable cure after completion of therapy. ${ }^{47}$ QTc prolongation and excess deaths in the bedaquiline groups of C208 small trials raise concerns about the safety of the drug. Drug interactions will limit utility of bedaquiline in patients receiving antiretroviral therapy. Excessive half-life will increase the risks to the patient when bedaquiline does cause toxicity and will increase the risks of the emergence of bedaquiline resistance if drugs are discontinued or have mismatched half-lives and long dosing intervals.

Particularly where MDR-TB and XDR-TB are highly prevalent, any new drug may be subjected to imperfect regimens and selective pressure. Until more novel drugs and regimens are developed, the use of bedaquiline must be carefully controlled so that drug resistance does not become widespread.
The development and marketing of bedaquiline, a member of a novel class of antituberculous drugs, is a landmark accomplishment for the tuberculosis community. Later generations of diarylquinolines may be more potent and less toxic and may be combined with other novel drugs that are in development to treat susceptible and drug-resistant tuberculosis with safe, effective, short-course regimens.

\section{Disclosure}

The authors report no conflicts of interest in this work.

\section{References}

1. Mitchison DA. The diagnosis and therapy of tuberculosis during the past 100 years. Am J Respir Crit Care Med. 2005;171(7):699-706.

2. Raviglione MC, Snider DE, Kochi A. Global epidemiology of tuberculosis. Morbidity and mortality of a worldwide epidemic. JAMA. 1995;273(3):220-226.

3. Dye C. Global epidemiology of tuberculosis. Lancet. 2006;367(9514): 938-940.

4. Lienhardt C, Glaziou P, Uplekar M, Lönnroth K, Getahun H, Raviglione M. Global tuberculosis control: lessons learnt and future prospects. Nat Rev Microbiol. 2012;10(6):407-416.

5. Rieder HL, Arnadottir T, Trébucq A, Enarson DA. Tuberculosis treatment: dangerous regimens? Int J Tuberc Lung Dis. 2001;5(1):1-3.

6. World Health Organization. Multidrug and extensively drug-resistant TB (M/XDR-TB): 2010 global report on surveillance and response. Geneva: World Health Organization; 2010. Available from: http://whqlibdoc. who.int/publications/2010/9789241599191_eng.pdf. Accessed June 13, 2014.

7. Gandhi NR, Moll A, Sturm AW, et al. Extensively drug-resistant tuberculosis as a cause of death in patients co-infected with tuberculosis and HIV in a rural area of South Africa. Lancet. 2006;368(9547):1575-1580.

8. Blumberg HM, Burman WJ, Chaisson RE, et al; American Thoracic Society, Centers for Disease Control and Prevention and the Infectious Diseases Society. American Thoracic Society/Centers for Disease Control and Prevention/Infectious Diseases Society of America: treatment of tuberculosis. Am J Respir Crit Care Med. 2003;167(4):603-662.

9. Iseman MD. Treatment of multidrug-resistant tuberculosis. $N$ Engl $J$ Med. 1993;329(11):784-791.

10. Law KF, Jagirdar J, Weiden MD, Bodkin M, Rom WN. Tuberculosis in HIV-positive patients: cellular response and immune activation in the lung. Am J Respir Crit Care Med. 1996;153(4 Pt 1):1377-1384.

11. Nakata K, Rom WN, Honda Y, et al. Mycobacterium tuberculosis enhances human immunodeficiency virus-1 replication in the lung. Am J Respir Crit Care Med. 1997;155(3):996-1003.

12. Abdool Karim SS, Naidoo K, Grobler A, et al. Integration of antiretroviral therapy with tuberculosis treatment. N Engl J Med. 2011;365(16): 1492-1501.

13. Blanc FX, Sok T, Laureillard D, et al; CAMELIA (ANRS 1295-CIPRA KH001) Study Team. Earlier versus later start of antiretroviral therapy in HIV-infected adults with tuberculosis. N Engl J Med. 2011;365(16): 1471-1481.

14. Havlir DV, Kendall MA, Ive P, et al; AIDS Clinical Trials Group Study A5221. Timing of antiretroviral therapy for HIV-1 infection and tuberculosis. N Engl J Med. 2011;365(16):1482-1491.

15. Burman WJ, Gallicano K, Peloquin C. Comparative pharmacokinetics and pharmacodynamics of the rifamycin antibacterials. Clinical Pharmacokinetics. 2001;40(5):327-341.

16. Managing Drug Interactions in the Treatment of HIV-Related Tuberculosis [webpage on the Internet]. Atlanta: Centers for Disease Control and Prevention; 2013. Available from: http://www.cdc.gov/tb/ TB_HIV_Drugs/default.htm. Accessed June 13, 2014. 
17. Nachega JB, Rosenkranz B, Simon G, Chaisson RE, Diacon A, Taljaard J. Management of adult active tuberculosis disease in era of HIV pandemic, current practices and future perspectives. Infect Disord Drug Targets. 2011;11(2):134-143.

18. Leistikow RL, Morton RA, Bartek IL, Frimpong I, Wagner K, Voskuil MI. The Mycobacterium tuberculosis DosR regulon assists in metabolic homeostasis and enables rapid recovery from nonrespiring dormancy. J Bacteriol. 2010;192(6):1662-1670.

19. Jindani A, Aber VR, Edwards EA, Mitchison DA. The early bactericidal activity of drugs in patients with pulmonary tuberculosis. Am Rev Respir Dis. 1980;121(6):939-949.

20. Boshoff HI, Barry CE 3rd. Tuberculosis - metabolism and respiration in the absence of growth. Nat Rev Microbiol. 2005;3(1):70-80.

21. Bennett DE, Courval JM, Onorato I, et al. Prevalence of tuberculosis infection in the United States population: the national health and nutrition examination survey, 1999-2000. Am J Respir Crit Care Med. 2008;177(3):348-355.

22. Glickman SW, Rasiel EB, Hamilton CD, Kubataev A, Schulman KA. Medicine. A portfolio model of drug development for tuberculosis. Science. 2006;311(5765):1246-1247.

23. TB Alliance Interactive Portfolio [webpage on the Internet]. Global Alliance for TB Drug Development. Available from: http://www. tballiance.org/portfolio/. Accessed February 21, 2014.

24. Andries K, Verhasselt P, Guillemont J, et al. A diarylquinoline drug active on the ATP synthase of Mycobacterium tuberculosis. Science. 2005;307(5707):223-227.

25. Haagsma AC, Abdillahi-Ibrahim R, Wagner MJ, et al. Selectivity of TMC207 towards mycobacterial ATP synthase compared with that towards the eukaryotic homologue. Antimicrob Agents Chemother. 2009;53(3):1290-1292.

26. Huitric E, Verhasselt P, Andries K, Hoffner SE. In vitro antimycobacterial spectrum of a diarylquinoline ATP synthase inhibitor. Antimicrob Agents Chemother. 2007;51(11):4202-4204.

27. Koul A, Vranckx L, Dendouga N, et al. Diarylquinolines are bactericidal for dormant mycobacteria as a result of disturbed ATP homeostasis. J Biol Chem. 2008;283(37):25273-25280.

28. Petrella S, Cambau E, Chauffour A, Andries K, Jarlier V, Sougakoff W. Genetic basis for natural and acquired resistance to the diarylquinoline R207910 in mycobacteria. Antimicrob Agents Chemother. 2006;50(8):2853-2856.

29. Gupta S, Cohen KA, Winglee K, Maiga M, Diarra B, Bishai WR. Efflux inhibition with verapamil potentiates bedaquiline in Mycobacterium tuberculosis. Antimicrob Agents Chemother. 2014;58(1):574-576.

30. Hartkoorn RC, Uplekar S, Cole ST. Cross-resistance between clofazimine and bedaquiline through upregulation of MmpL5 in Mycobacterium tuberculosis. Antimicrob Agents Chemother. 2014;58(5):2979-2981.

31. Janssen Pharmaceutical Companies. Anti-Infective Drugs Advisory Committee Meeting Briefing Document TMC207 (bedaquiline) Treatment of Patients with MDR-TB NDA 204-384. Titusville, NJ: Janssen Pharmaceutical Companies; 2012. Available from: http://www. fda.gov/downloads/advisorycommittees/committeesmeetingmaterials/ drugs/anti-infectivedrugsadvisorycommittee/ucm329260.pdf. Accessed February 21, 2014.

32. Nuermberger E, Mitchison DA. Once-weekly treatment of tuberculosis with the diarylquinoline R207910: a real possibility. Am J Respir Crit Care Med. 2009;179(1):2-3.

Therapeutics and Clinical Risk Management

\section{Publish your work in this journal}

Therapeutics and Clinical Risk Management is an international, peerreviewed journal of clinical therapeutics and risk management, focusing on concise rapid reporting of clinical studies in all therapeutic areas, outcomes, safety, and programs for the effective, safe, and sustained use of medicines. This journal is indexed on PubMed Central, CAS,
33. Veziris N, Ibrahim M, Lounis N, et al. A once-weekly R207910-containing regimen exceeds activity of the standard daily regimen in murine tuberculosis. Am J Respir Crit Care Med. 2009;179(1): 75-79.

34. Rustomjee R, Diacon AH, Allen J, et al. Early bactericidal activity and pharmacokinetics of the diarylquinoline TMC207 in treatment of pulmonary tuberculosis. Antimicrob Agents Chemother. 2008;52(8): 2831-2835.

35. Diacon AH, Pym A, Grobusch M, et al. The diarylquinoline TMC207 for multidrug-resistant tuberculosis. $N$ Engl J Med. 2009;360(23): 2397-2405.

36. Barry CE 3rd. Unorthodox approach to the development of a new antituberculosis therapy. $N$ Engl J Med. 2009;360(23):2466-2467.

37. Diacon AH, Dawson R, Von Groote-Bidlingmaier F, et al. Randomized dose-ranging study of the 14-day early bactericidal activity of bedaquiline (TMC207) in patients with sputum microscopy smear-positive pulmonary tuberculosis. Antimicrob Agents Chemother. 2013;57(5): 2199-2203.

38. Lounis N, Veziris N, Chauffour A, Truffot-Pernot C, Andries K, Jarlier V. Combinations of R207910 with drugs used to treat multidrug-resistant tuberculosis have the potential to shorten treatment duration. Antimicrob Agents Chemother. 2006;50(11):3543-3547.

39. Diacon AH, Donald PR, Pym A, et al. Randomized pilot trial of eight weeks of bedaquiline (TMC207) treatment for multidrug-resistant tuberculosis: long-term outcome, tolerability, and effect on emergence of drug resistance. Antimicrob Agents Chemother. 2012;56(6): 3271-3276.

40. Phase 2 open-label trial of TMC207 in an MDR-TB treatment regimen [webpage on the Internet]. Paris: International Union Against Tuberculosis and Lung Disease; 2011. Available from: http://uwclh. conference2web.com/content/1108. Accessed February 21, 2014.

41. Anti-Infective Drugs Advisory Committee Meeting [webpage on the Internet]. Silver Spring, MD: US Food and Drug Administration; 2012. Available from: http://www.fda.gov/downloads/AdvisoryCommittees/ CommitteesMeetingMaterials/Drugs/Anti-InfectiveDrugsAdvisoryCommittee/UCM332961.pdf. Accessed February 21, 2014.

42. Accelerated Approval Program [webpage on the Internet]. Silver Spring, MD: US Food and Drug Administration; 2013. Available from: http://www.fda.gov/Drugs/ResourcesForYou/HealthProfessionals/ ucm313768.htm. Accessed February 21, 2014.

43. Sirturo ${ }^{\mathrm{TM}}$ [prescribing information]. Titusville, NJ: Janssen Pharmaceutical Companies; 2012.

44. Janssen Infectious Diseases BVBA. A study to evaluate the efficacy and safety of TMC207 in patients with pulmonary infection with multi-drug resistant Mycobacterium tuberculosis. Available from: http://clinicaltrials.gov/ct2/show/NCT01600963?term=bedaquiline \&rank=7. NLM identifier: NCT01600963. Accessed February 21, 2014

45. Centers for Disease Control and Prevention. Provisional CDC guidelines for the use and safety monitoring of bedaquiline fumarate (Sirturo) for the treatment of multidrug-resistant tuberculosis. MMWR Recomm Rep. 2013;62(RR-09):1-12.

46. Tasneen R, Li SY, Peloquin CA, et al. Sterilizing activity of novel TMC207- and PA-824-containing regimens in a murine model of tuberculosis. Antimicrob Agents Chemother. 2011;55(12):5485-5492.

47. Avorn J. Approval of a tuberculosis drug based on a paradoxical surrogate measure. JAMA. 2013;309(13):1349-1350.

\section{Dovepress}

EMBase, Scopus and the Elsevier Bibliographic databases. The manuscript management system is completely online and includes a very quick and fair peer-review system, which is all easy to use. Visit http://www.dovepress.com/testimonials.php to read real quotes from published authors. 